\title{
Usefulness of a Diluted Prothrombin Time for Accurately Diagnosing Antiphospholipid Syndrome
}

\author{
Keiji Nakai ${ }^{1}$, Hideo Wada ${ }^{2, *}$, Kaname Nakatani ${ }^{2}$, Yuko Kamikura ${ }^{3}$, Takeshi Mastumoto ${ }^{3}$, \\ Toshihiko Kobayashi ${ }^{3}$, Harue Tonomura ${ }^{2}$, Yasutaka Tono ${ }^{2}$, Mariko Ohyabu ${ }^{2}$, Satoshi Ota ${ }^{3}$, \\ Norikazu Yamada ${ }^{4}$, Yuri Besho ${ }^{5}$, Eri Yamada ${ }^{5}$, Makoto Ikejiri $^{5}$, Yasunori Abe $^{5}$ and \\ Tsutomu Nobori ${ }^{2}$
}

\author{
${ }^{1}$ Medical Education Development Center, ${ }^{2}$ Department of Molecular and Laboratory Medicine, ${ }^{3}$ Department of Hema- \\ tology and Oncology, ${ }^{4}$ Department of Cardiology, ${ }^{5}$ Centeral Laboratory, Mie University Graduate School of Medicine, \\ Tsu, Mie, Japan
}

\begin{abstract}
The usefulness of lupus anticoagulant (LA) and several antibodies for predicting thrombosis was assessed in patients with idiopathic thrombocytopenic purpura (ITP), systemic lupus erythematosus (SLE), lupus like disease (LLD), recurrent abortion and primary antiphospholipid syndrome (APS). LA was measured using the diluted Russell Viper Venom test (DRVVT) and the diluted prothrombin time (dPT).

In healthy volunteers, the median (range) of the DRVVT and dPT ratio were 0.97 (0.88 - 1.08) and $1.05(0.85-1.29)$, respectively. The positive percent of dPT ratio was high in patients with ITP, SLE, recurrent abortion and primary APS. The sensitivity for thrombosis was highest for the dPT ratio and the specificity for thrombosis was highest for the DRVVT ratio. The sensitivity and the Odd's ratio for both the dPT and DRVVT ratio were high. A positive predictive value in DRVVT and a negative predictive value in dPT were high. The receiver operating characteristic (ROC) curve analysis indicates that the dPT ratio might be more useful for predicting thrombosis than the DRVVT ratio. The DRVVT and dPT ratios are useful for both the diagnosis of APS as well as predicting thrombosis.
\end{abstract}

Keywords: Diluted prothrombin time, antiphospholipid syndrome, diluted russell viper venom test, lupus anticoagulant.

\section{INTRODUCTION}

Patients with Antiphospholipid syndrome (APS) are subject to thrombosis in arteries and/or veins, pregnancy loss, and thrombocytopenia, associated with antiphospholipid antibodies [1, 2]. Anti-phospholipid antibodies (APA) include lupus anticoagulant (LA), anti-cardiolipin (aCL), antiprothrombin (aPT) and anti- $\beta 2$-glycoproteinI (anti- $\beta 2$-GPI) antibodies [3-6]. Lupus anticoagulants (LA) are considered to be immunoglobulins which are directed to either prothrombin or $\beta 2$-GPI bound to phospholipids and which are highly sensitive for APS [7]. Almost all patients with APS have either $\beta 2$-GPI- or prothrombin-dependent antibodies. Therefore, accurate detection of LA is important in patients suspected of having APS. The combination of the dilute activated partial thromboplastin time (dAPTT) and the dilute Russell viper venom time (DRVVT)-based LA tests are a simple and reproducible way of detecting the presence of LAs with a high sensitivity and specificity [8]. The dilute prothrombin time (dPT) is a well documented screening test for LA [9]. However, when using a crude mixture of tissue factor and PL, it tends to have a poor reproducibility and

*Address correspondence to this author at the Department of Molecular and Laboratory Medicine, Mie University Graduate School of Medicine, 2-174 Edobashi, Tsu, Mie 514-8507, Japan; Tel: 81-59-232-1111; Fax: 81-59-2315204; E-mail wadahide@clin.medic.mie-u.ac.jp specificity [10]. Arnout et al. [9] showed that the dPT performed with human tissue thromboplastin obtained by recombinant DNA technology considerably improved the test results.

In this study, we investigated the usefulness of dPT for the detection of LA in addition to DRVVT.

\section{MATERIALS AND METHODS}

Several APA were examined in 100 patients with suspected of having LA and consisting of 28 men and 72 women ranging in age from 35 to 59.5 years, with a mean age of 47.0. The underlying diseases of the patients were as follows; 40 idiopathic thrombocytopenic purpura (ITP), 34 lupus like disease (LLD), 12 systemic lupus erythematosus (SLE), 6 habitual pregnancy loss, 5 primary antiphospholipid syndrome (primary APS) and 3 hemophilia. The presence of recurrent arterial and venous thrombosis, pregnancy complications of 100 patients were as follows; 10 had brain thrombosis, 7 had deep vein thrombosis (DVT)/ pulmonary embolism (PE), 2 had central retinal artery occlusion (CRVO), 6 had habitual miscarriage and 2 had other diseases.

DRVVT was measured using a DVV-test (American Diagnostica Inc., ADI, Stamford, CT, USA) and DVV-confirm (ADI). The patients who have more than 1.2 of DVV-test/ 
DVV ratio are considered as LA positive. dPT was measured using a ACTICLOT dPT (ADI). A low lipid concentrated PT/ high lipid concentrated PT ratio of higher than 1.3 was considered as LA positive. The cutoff and the mean value for the DRVVT and dPT ratios were examined in 60 healthy volunteers consisting of 43 men and 17 women ranging in age from 21 to 54 years old, and the mean age was 31.5 years old.

Anti-phospholipid (aPL) antibody, aPT antibody or anti$\beta 2 \mathrm{GP} 1$ antibody was measured using an enzyme-linked immunosorbent assay (ELISA) using the IMUCLONE aPL IgG ELISA Kit (ADI), the IMUCLONE Anti-Prothrombin IgG

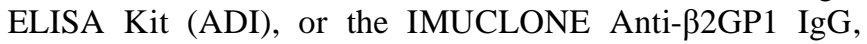
ELISA Kit (ADI).

\section{Statistical Analysis}

The data are expressed as the mean \pm SD or median; range. Differences between the groups were examined for significance using the Mann-Whitney $U$ test while any correlations between the 2 variables were tested by Pearson's correlation analysis. A $\mathrm{P}$ value $<0.05$ was considered significant. The usefulness of D-dimer and soluble fibrin (SF) for the diagnosis of thrombosis, DVT and disseminated intravascular coagulation (DIC) was examined by a receiver operating characteristic (ROC) analysis. The cutoff values were determined by an ROC analysis. All statistical analyses were performed using the SPSS II software package (SPSS Japan, Tokyo).

\section{RESULTS}

Fig. (1) shows the DRVVT and dPT ratio in healthy volunteers and those who do not demonstrate a normal distribution. The median (range) of the DRVVT and dPT ratios were $0.97(0.88-1.08)$ and $1.05(0.85-1.29)$, respectively. Next, the cutoff value was determined to be 1.2 for the DRVVT ratio and 1.3 for the $\mathrm{dPT}$ ratio.
The positive percentage of the dPT ratio (more than 1.3) and DRVVT ratio (more than 1.2) was $45.0 \%$ and $17.5 \%$ in patients with ITP, $23.5 \%$ and $23.5 \%$ in those with LLD, $66.7 \%$ and $66.7 \%$ in those with SLE, $83.3 \%$ and $16.7 \%$ in those with recurrent abortion, $80.0 \%$ and $20.0 \%$ in those with primary APS, and $0.0 \%$ and $0.0 \%$ in those with hemophilia, respectively (Table 1). The positive percentage of aPT and anti- $\beta 2$ GP1 antibody were high in the patients with SLE but low in the other groups. The positive percentage of aPL was low in all groups.

The dPT ratio (median; range) was significantly higher in the patients with thrombosis $(1.37 ; 1.27-1.56)$ than in those without thrombosis $(1.12 ; 1.01-1.30)$ but not the DRVVT ratio (Fig. 2). The sensitivity for thrombosis was the highest for the dPT ratio $(92.6 \%)$ while the specificity for thrombosis was highest for the DRVVT ratio $(82.2 \%$ ) (Table 2 ).

An ROC analysis shows the dPT ratio to be more useful for the diagnosis of thrombosis than the DRVVT ratio (Fig. 3). These data indicated the adequate cutoff value for thrombosis to be 1.09 for the DRVVT ratio and 1.26 for the dPT ratio. At this cutoff value, the sensitivity and Odd's ratio for both the dPT and DRVVT ratios were high, while the positive predictive value (PPV) for the DRVVT ratio and the negative predictive value (NPV) for the dPT ratio were high (Table 3).

\section{DISCUSSIONS}

From the data obtained in healthy volunteers, the cutoff values for DRVVT and dPT ratios were 1.2 and 1.3, respectively. The value of DRVVT was similar to that previously described $[7,11]$. The positive percentage of the dPT ratio was high in ITP, SLE, recurrent abortion and primary APS. The frequency of LA was the highest in collagen diseases. Previously, APS has been frequently reported in collagen diseases $[8,10]$. As LA was observed in both patients with ITP and SLE without thrombosis and healthy volunteers, LA

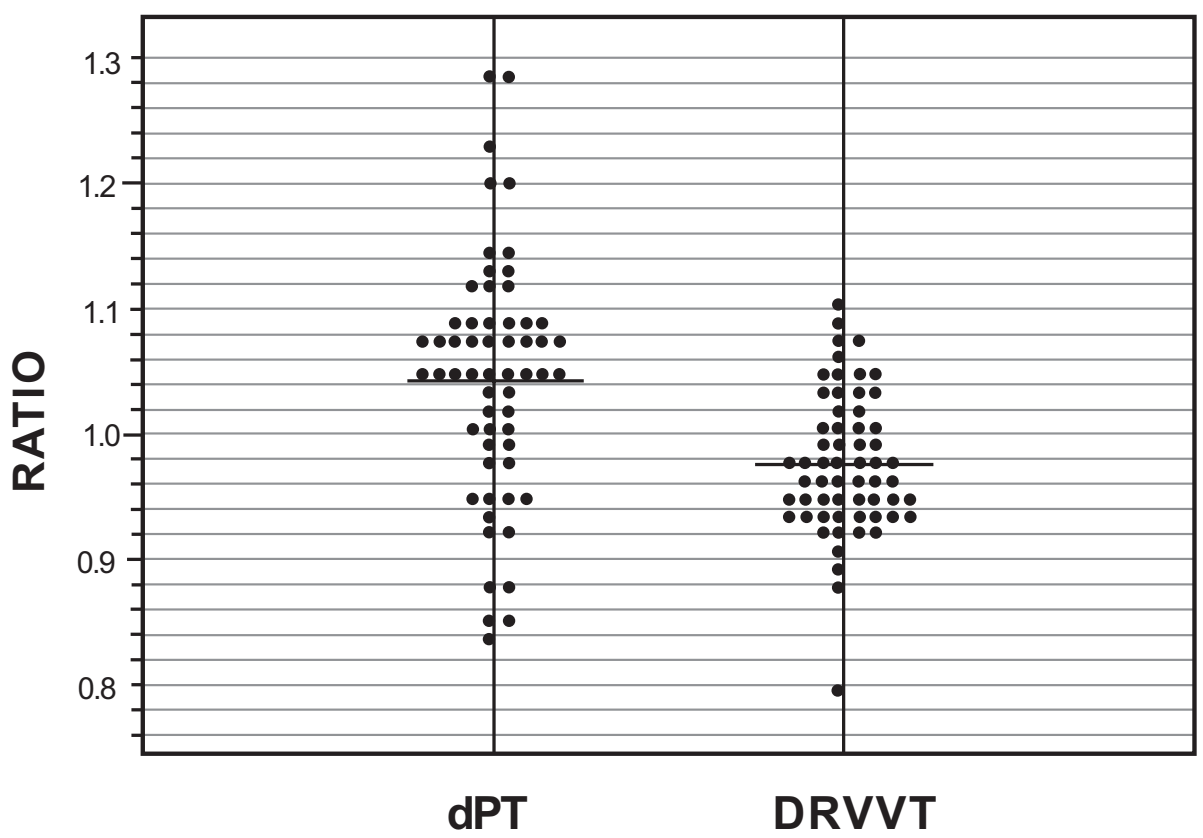

Fig. (1). The dilute prothrombin time (dPT) and diluted Russell Viper Venom test (DRVVT) ratios in healthy volunteers. 
Table 1. Positive Percent of Anti-Phospholipid Antibody in Various Diseases

\begin{tabular}{|c|c|c|c|c|c|c|}
\hline & ITP & LLD & SLE & Abortion & PAPS & Hemophilia \\
\hline \hline aPT & $12.5 \%$ & $6.7 \%$ & $41.7 \%$ & $16.7 \%$ & $0 \%$ & $0 \%$ \\
\hline Anti- $\beta 2 \mathrm{GPI}$ & $12.5 \%$ & $8.8 \%$ & $50.0 \%$ & $33.3 \%$ & $16.7 \%$ & $20 \%$ \\
\hline aPL & $0 \%$ & $3.3 \%$ & $27.3 \%$ & $83.3 \%$ & $80 \%$ & $0 \%$ \\
\hline dPT ratio & $45.0 \%$ & $23.5 \%$ & $66.7 \%$ & $16.7 \%$ & $40 \%$ & $0 \%$ \\
\hline DRVVT ratio & $17.5 \%$ & $23.5 \%$ & $66.7 \%$ & & $0 \%$ \\
\hline
\end{tabular}

aPT: anti-prothrombin, anti- $\beta 2$-GPI: anti- $\beta 2$-glycoprotein I, aPL: Anti-phospholipid, dPT: dilute prothrombin time, DRVVT: diluted Russell Viper Venom test, ITP: idiopathic thrombocytopenic purpura, LLD: lupus like disease, SLE: systemic lupus erythematosus, PAPS: primary anti-phospholipid syndrome.

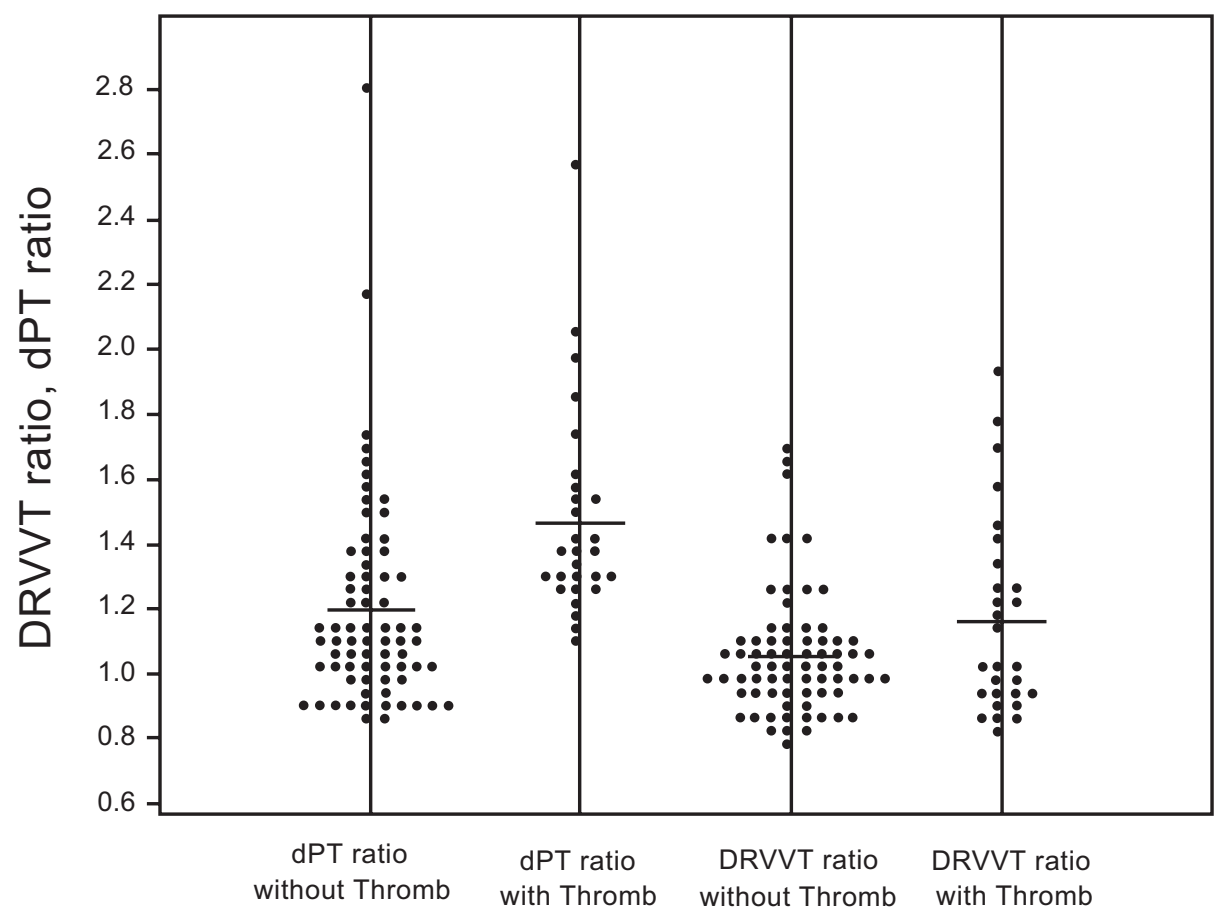

Fig. (2). The dilute prothrombin time (dPT) and diluted Russell Viper Venom test (DRVVT) ratios in patients with or without thrombosis $* *: \mathrm{p}<0.01$

Thromb: thrombosis

is not always a risk factor for thrombosis. LA was also reported in other patients without thrombosis $[12,13]$. Since a positive frequency was higher for the dPT ratio than for the DRVVT ratio, the dPT ratio was thus suggested to possibly be a sensitive assay for LA.

Table 2 Sensitivity and Specificity of Anti-Phospholipid Antibody for Thrombosis Based on the Cutoff Values from Healthy Volunteers

\begin{tabular}{|c|c|c|}
\hline & Sensitivity & Specificity \\
\hline \hline dPT ratio & $92.6 \%$ & $75.3 \%$ \\
\hline DRVVT ratio & $48.1 \%$ & $82.2 \%$ \\
\hline
\end{tabular}

dPT: dilute prothrombin time, DRVVT: diluted Russell Viper Venom test, Cutoff value: $\mathrm{dPT}$ ratio; 1.30 , DRVVT ratio; 1.20.
In other antibodies, the positive percent of aPT and anti$\beta 2 \mathrm{GP} 1$ antibody were high in patients with SLE but low in the other groups. The positive percent of aPL was low in all groups. The frequency of anti- $\beta 2 \mathrm{GP} 1 \mathrm{IgG}$ was markedly high in patients with cerebral thrombosis but only slightly high in patients with DVT, thus suggesting this frequency to be related to thrombosis, especially arterial thrombosis [7]. Cerebral ischemia associated with anti-phospholipid antibody is the most common arterial thrombotic manifestation in APS [14, 15]; however, the importance of antiphospholipids as a cardiovascular risk factor remains controversial. The frequency of aPT IgG antibody was moderately high in patients with DVT and low in those with cerebral thrombosis and SLE, thus suggesting that it is related to thrombosis, especially venous thrombosis [7]. Prothrombin, another phospholipid binding protein, was first proposed as a possible cofactor for LA by Loeliger in 1959 [16]. A positive correlation between the presence of aPT antibody and DVT 
Table 3. Sensitivity and Specificity of the DRVVT and dPT Ratios for Thrombosis Based on a Cutoff Value from an ROC Analysis

\begin{tabular}{|c|c|c|}
\hline & dPT Ratio & DRVVT Ratio \\
\hline \hline Cutoff value & 1.26 & 7.09 \\
\hline Sensitivity & $75.0 \%$ & $67.9 \%$ \\
\hline Specificity & $70.8 \%$ & $85.5 \%$ \\
\hline PPV & $50.0 \%$ & $50.0 \%$ \\
\hline NPV & $87.9 \%$ & 5.89 \\
\hline Odd's ratio & 7.29 & 0.702 \\
\hline AUS & 0.794 & \\
\hline
\end{tabular}

dPT: dilute prothrombin time, DRVVT: diluted Russell Viper Venom test,

PPV: positive predictive value, NPV: negative predictive value

AUC: area under the curve

Cutoff value: dPT ratio; 1.26 , DRVVT ratio; 1.09

was reported in a SLE population [17]. In a study of 265 cases of DVT or PE, the risk of thrombotic events was shown to significantly increase in carriers of aPT antibody [18].

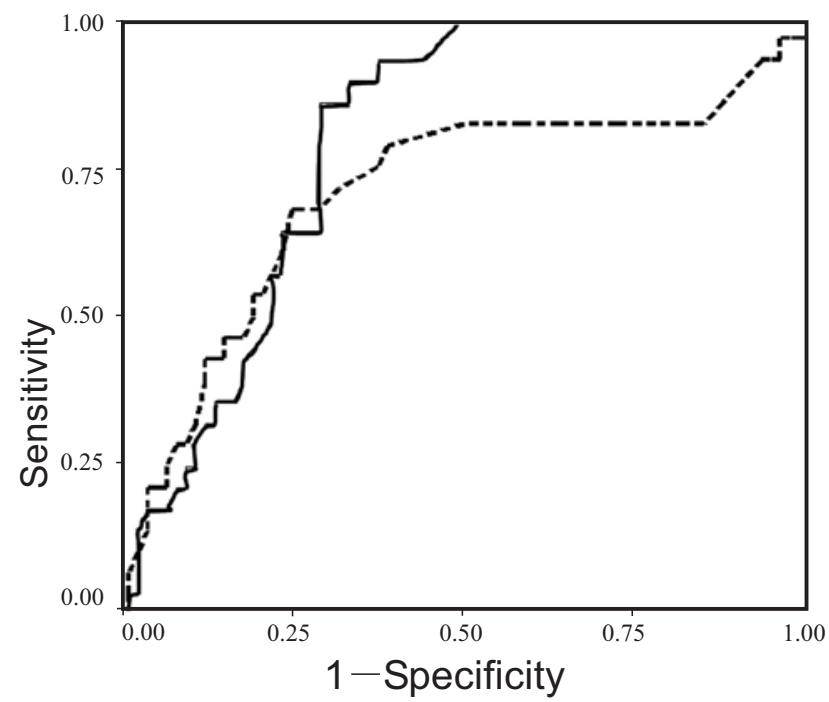

Fig. (3). Receiver operating characteristic (ROC) analysis of dilute prothrombin time (dPT) and diluted Russell Viper Venom test (DRVVT) ratio for thrombosis

-dPT ratio, ----- DRVVT ratio

The sensitivity for thrombosis was the highest for the dPT ratio and the specificity for thrombosis was the highest for the DRVVT ratio, thus suggesting that a lightly lower cutoff value of the DRVVT ratio might thus be better for diagnosing of thrombosis. Indeed, an adequate cutoff value of the DRVVT ratio for thrombosis from an ROC analysis was slightly low; the sensitivity and Odd's ratio for both the dPT and DRVVT ratios were high, the PPV for the DRVVT ratio and the NPV for the APT ratio were high. An ROC analysis and Odd's ratio show that the dPT might therefore be more useful for predicting thrombosis than DRVVT.
APA is reported to interfere with tissue factor pathway inhibitor (TFPI), which is an important inhibitor for the extrinsic pathway of blood coagulation [19]. Especially, TFPI regulates the inhibitory effect of blood coagulation on the phospholipid surface [20]. Therefore, a dPT assay may be a sensitive tool for diagnosing thrombosis.

\section{ACKNOWLEDGEMENTS}

This work was supported in part by research grants from the Japanese Ministry of Health and Welfare and Japanese Ministry of Education Culture and Science.

\section{REFFERENCES}

[1] Harris EN. Antiphospholipid syndrome. In: Klippel JH, Dieppe PA, Eds. Rheumatology, London: Mosby-Year Book, 1994; Section 6, 32.1-32.6.

[2] Harris EN, Gharavi AE, Hughes GRV. Antiphospholipid antibodies. Clin Rheum Dis 1985; 11: 591-601.

[3] Ginsberg I, Wells P, Brill-Edwards P, et al. Antiphospholipid antibodies and venous thromboembolism. Blood 1995; 86: 3685-91.

[4] Kearon C, Gent M, Hirsh J, et al. A comparison of three months of anticoagulation with extended anticoagulation for a first episode of idiopathic venous thromboembolism. N Engl J Med 1999; 340: 901-07.

[5] Schulman S, Svenungsson E, Granqvist S, the Duration of Anticoagulant Study Group. Anticardiolipin antibodies predict early recurrence of thromboembolism and death among patients with venous thromboembolism following anticoagulant therapy. Am J Med 1998; 104: 332-8

[6] Simioni P, Prandoni P, Zanon E, et al. Deep vein thrombosis and lupus anticoagulant. A case-control study. Thromb Haemost 1996; 76: $187-9$.

[7] Ota S, Wada H, Nobori T, et al. Diagnosis of deep vein thrombosis by plasma-soluble fibrin or D-dimer. Am J Hematol 2005; 79: 27480.

[8] Ghirardello A, Doria A, Ruffatti A, et al. Antiphospholipid antibodies (aPL) in systemic lupus erythematosus. Are they specific tool for the diagnosis of aPL syndrome. Ann Rheum Dis 1994; 53: 140-2.

[9] Arnout J, Vanrusselt M, Huybrechts E, Vermylen J. Optimization of the dilute prothrombin time for the detection of the lupus anticoagulant by use of a recombinant tissue thromboplastin. $\mathrm{Br} \mathrm{J}$ Haematol 1994; 87: 94-9.

[10] Horbach DA, van Oort E, Donders RC, et al. Lupus anticoagulant is the strongest risk factor for both venous and arterial thrombosis in patients with systemic lupus erythematosus - comparison be- 
tween different assays for the detection of antiphospholipid antibodies. Thromb Haemost 1996; 76: 916-24.

[11] Nencini P, Baruffi M, Abbate R, Amaducci L, Inzitari D. Lupus anticoagulant and anti-cardiolipin antibodies in young adults with cerebral ischemia. Stroke 1992; 23: 189-93.

[12] Nakase T, Wada H, Minamikawa $\mathrm{K}$, et al. Increased activated protein $\mathrm{C}$ - protein $\mathrm{C}$ inhibitor complex level in patients positive for lupus anticoagulant. Blood Coagul Fibrinolysis 1994; 5: 173-7.

[13] Sakakura M, Wada H, Watanabe R, et al. Coagulation tests and anti-phospholipid antibodies in patients positive for lupus anticoagulant. Clin Appl Thromb Haemost 2000; 6: 144-50.

[14] Shah NM, Khamashta MA, Atsumi T, Hughes GRV. Outcome of patients with anticardiolipin antibodies: a 10 year follow-up of 52 patients. Lupus 1998; 7: 3-6.

[15] Krnic-Barrie S, Reister O'Connor C, Looney SW, Pierangeli SS, Harris EN. A retrospective review of 61 patients with antiphospholipid syndrome. Arch Intern Med 1997; 157: 2101-08.
[16] Loeliger A. Prothrombin as a co-factor of the circulating anticoagulant in systemic lupus erythematosus? Thromb Diath Haemorrh 1959; 3: 237-56.

[17] Puurunen M, Vaarala O, Julkunen H, Aho K, Palosuo T. Antibodies to phospholipid-binding plasma proteins and occurrence of thrombosis in patients with systemic lupus erythematosus. Clin Immunol Immunopathol 1996; 80: 16-22.

[18] Palosuo T, Virtamo J, Haukka J, et al. High antibody levels to prothrombin imply a risk of deep venous thrombosis and pulmonary embolism in middle-aged men. A nested case-control study. Thromb Haemost 1997; 78: 1178-82.

[19] Jacobsen EM, Sandset PM, Wisloff F. Do antiphospholipid antibodies interfere with tissue factor pathway inhibitor? Thromb Res 1999; 94: 213-20.

[20] Lindhout T, Salemink I, Valentin S, Willems GM. Tissue factor pathway inhibitor: regulation of its inhibitory activity by phospholipid surfaces. Haemostasis 1996; 26 (Suppl) 4: 89-97.

(C) Nakai et al.; Licensee Bentham Open.

This is an open access article licensed under the terms of the Creative Commons Attribution Non-Commercial License (http://creativecommons.org/licenses/by-nc/3.0/) which permits unrestricted, non-commercial use, distribution and reproduction in any medium, provided the work is properly cited. 